\title{
Seminoma de testículo abdominal en un paciente adulto: reporte de un caso
}

\author{
Cristián Palma C*, Cristóbal B, Maccioni R. \\ *Servicio de Urología del Hospital Clínico Universidad de Chile y Hospital San José. Santiago, Chile.
}

Actas Urol Esp. 2007;31(2):160-163

\section{RESUMEN}

SEMINOMA DE TESTÍCULO ABDOMINAL EN UN PACIENTE ADULTO: REPORTE DE UN CASO

Objetivo: Presentar un nuevo caso de Seminoma de Testículo Abdominal en un paciente adulto y una revisión de la literatura.

Observación clínica: Presentamos a un paciente de 30 años con criptorquidia, que luego de consultar por dolor abdominal en el servicio de urgencias, es diagnosticado un Seminoma de testículo abdominal.

Discusión: La incidencia de cáncer testicular en testículos no descendidos es de 3 a 48 veces mayor que en la población general. La existencia de testículos no descendidos en la población adulta es rara en los países desarrollados debido a la práctica sistemática de orquidopexia electiva antes de los 2 años de vida y orquiectomía en pacientes postpuberes con testículos no descendidos. A pesar de estas medidas de prevención, aún se encuentran casos aislados de tumores testiculares abdominales en adultos.

Palabras clave: Tumor testicular. Seminoma. Criptorquidia.

\section{ABSTRACT \\ NEW CASE OF AN INTRA-ABDOMINAL TESTICULAR SEMINOMA IN AN ADULT}

Objective: To present a new case of an Intra-abdominal Testicular Seminoma in an adult.

Methods/results: We report a 30 year old patient with cryptochidism, that after consulting to the emergency service due to abdominal pain, was diagnosed an Intra-abdominal Testicular Seminoma.

Discussion: The incidence of testicular cancer in cryptorchidic testis is 3 to 48 times higher than in general population. The existence of maldescended testis in adult population is rare in developed countries, due to the normal practice of elective orchidopexia in children under 2 years old and orchiectomy in maldecended testis in postpuberal pacients. In spite these measures of prevention, there are still isolated cases of Intra-abdominal testicular tumors in adults.

Keywords: Testicular tumor. Seminoma. Cryptochidism.

$\mathrm{L}$ a criptorquidia es una patología muy frecuente que afecta al 2-5\% de los niños ${ }^{1}$. En testículos criptorquideos la incidencia de cáncer testicular se estima entre 3 a 48 veces mayor al de la población general. El cáncer testicular se desarrolla en el $10 \%$ de los casos sobre testículos no descendidos ${ }^{2,3}$. Entre los testículos no descendidos, es mas frecuente el cáncer en testículos abdominales (30\% de los casos), que en los localizados en el canal inguinal ${ }^{4}$. Debido a las medi- das de prevención instauradas, actualmente es raro encontrar casos de tumores en testículos abdominales. Esta nota clínica apunta a presentar un nuevo caso de un paciente adulto con un tumor testicular intraabdominal.

\section{CASO CLÍNICO}

Presentamos un paciente de sexo masculino de 30 años de edad que consulta por dolor abdominal en el Servicio de Urgencia del Hospital San 
José, Santiago de Chile. El examen físico revela una masa palpable en fosa iliaca derecha. Destaca además a la palpación, la ausencia del testículo derecho. La ecografia demuestra una masa heterogénea sugerente de un absceso encapsulado. Se complementa con una tomografía axial computada en la cual se observa la presencia de una masa sólida de aproximadamente $10 \times 8 \times 7 \mathrm{cms}$. que ocupa el flanco y la fosa ilíaca derecha. Se constata además la presencia de extensas adenopatías retroperitoneales y mesentéricas. La radiografía de tórax fue normal. Los marcadores tumorales sólo revelan una discreta alza de la subunidad beta de la gonadotrofina coriónica (ß-HCG).

El paciente fue sometido a una laparotomía confirmándose el diagnóstico de un tumor testicular intraabdominal. La resección fue llevada a cabo sin incidentes. El análisis histopatológico fue compatible con un cáncer infiltrante de testículo, tipo histológico Seminoma clásico. El peso fue de 516 gramos y las dimensiones del tumor de 14,5 x 9,5 x 6,5 cms. Presentó además compromiso del epidídimo y la túnica albugínea en forma focal. Los marcadores tumorales postoperatorios resultaron dentro de rangos normales. El paciente fue derivado con indicación de Quimioterapia (Figs. 1, 2, 3 y 4).

\section{DISCUSIÓN}

El tumor testicular representa el 1-2\% de todas las neoplasias del sexo masculino y el $4 \%$

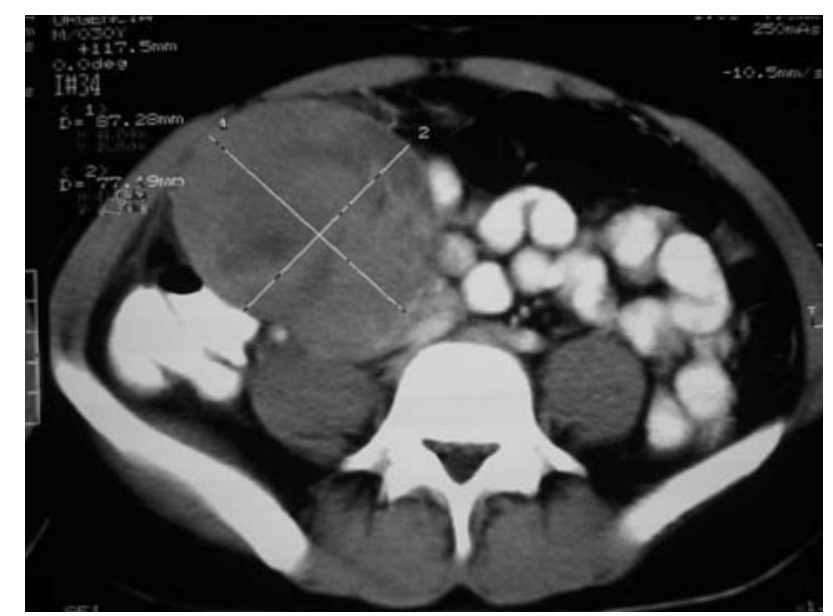

FIGURA 1. TAC: Masa sólida de aprox. $10 \times 8 \times 7 \mathrm{~cm}$. en fosa iliaca y flanco derecho. Extensas adenopatias retroperitoneales y mesentéricas.

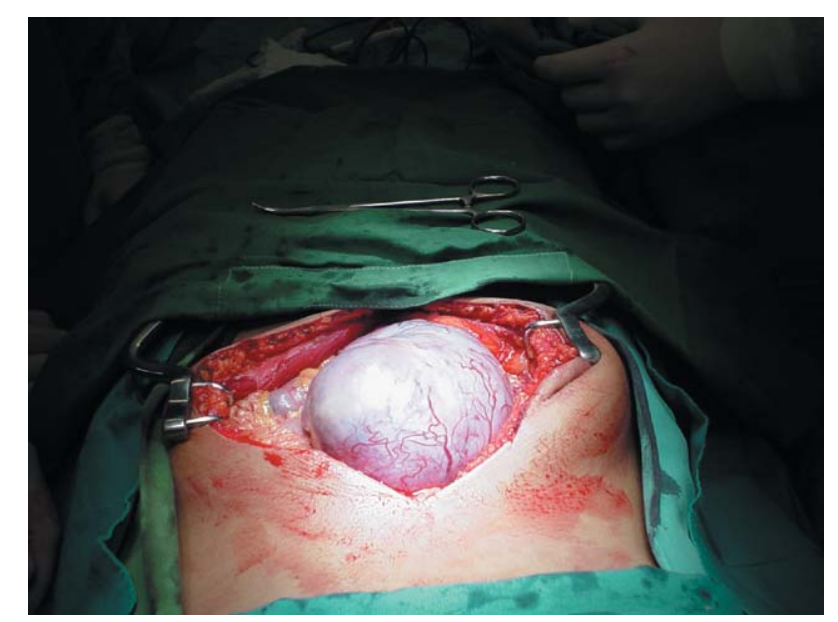

FIGURA 2. Paciente durante la cirugía de resección del tumor testicular intraabdominal.

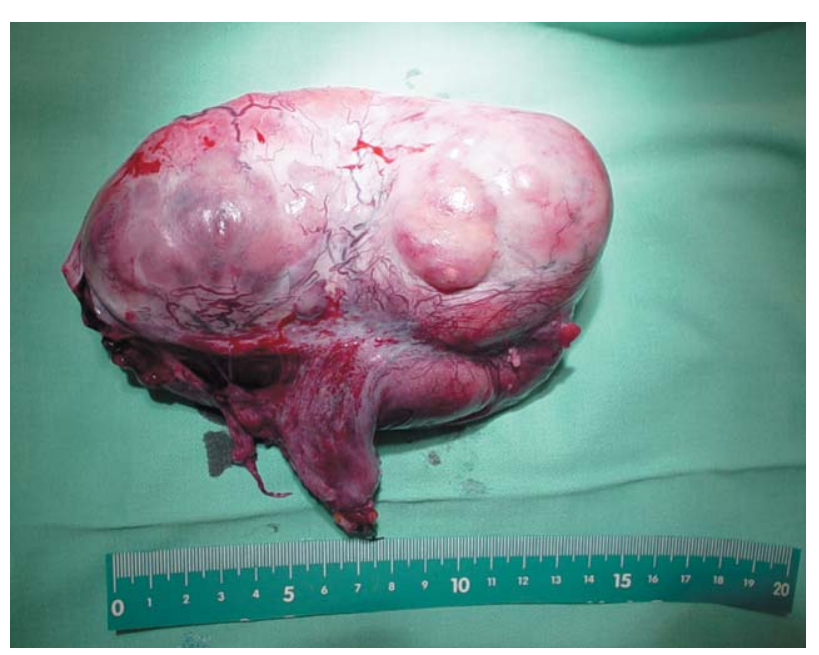

FIGURA 3. Aspecto macroscópico de la pieza después de realizar la orquiectomía. Peso de 516 grs. y dimensiones de $14,5 \times 9,5 \times 6,5$ cms.

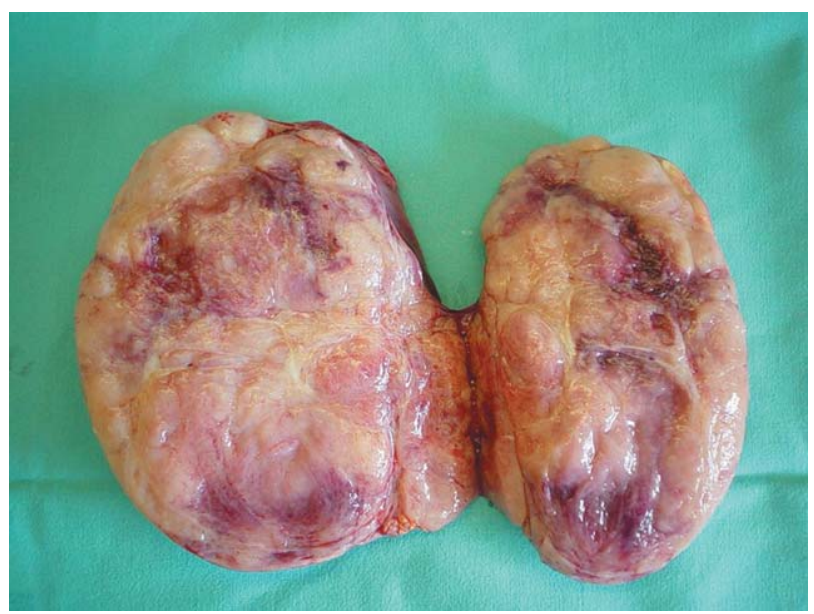

FIGURA 4. Anatomía patológica: Seminoma clásico con compromiso del epididimo y túnica albugínea. 
de las urogenitales. Es el tumor sólido mas frecuente en varones jóvenes entre los 20-35 años suponiendo del 1-2\% del total de tumores ${ }^{5}$. Hay muchos factores etiológicos propuestos en el desarrollo del cáncer testicular: traumatismos, atrofia testicular, disgenesia gonadal, siendo la criptorquidia y el antecedente de tumor en el testículo contralateral los más significativos ${ }^{6,7}$. En testículos criptorquideos la incidencia de cáncer testicular se estima entre 3 a 48 veces mayor al de la población general ${ }^{1,2}$. Aproximadamente un $10 \%$ de todos los tumores testiculares aparecen sobre un testículo no descendido ${ }^{1,2}$.

La criptorquidia es una patología muy frecuente que afecta al $2-5 \%$ de los niños. Los testículos no palpables corresponden a un $20 \%$ de los testículos criptorquideos. De los testículos no palpables, sólo en el $20 \%$ de los casos está ausente, el resto se encuentra en el abdomen o en el canal inguinal. Entre los testículos no descendidos, los testículos abdominales presentan una tasa más alta de malignización que los localizados en el canal inguinal ${ }^{1}$. Los testículos abdominales desarrollan un cáncer en el 30\% de los casos $^{4}$.

Las altas temperaturas en el canal inguinal y abdomen serían los responsables de la degeneración maligna del órgano ${ }^{1}$. Es por estas razones que en los pacientes con testículos no palpables, se debe localizar el testículo. Si el pacientes es postpuberal se recomienda la orquiectomía, especialmente por debajo de los 32 años ${ }^{8}$. Algunos autores realizan una laparoscopia exploradora, que es diagnóstica como terapéutica a la vez para la mayoría de los $\operatorname{casos}^{9}$.

Los tumores en testículos no descendidos en niños son raros, generalmente se diagnostican por una torsión del pediculo e histopatológicamente corresponden a un teratoma ${ }^{10}$. En cambio en adultos es mas frecuente encontrar tumores germinales y de estos el más frecuente es el Seminoma. La histopatología de los tumores de testículo no descendido en el adulto depende de la localización, siendo la proporción de Seminoma puro de 93\% cuando se encuentra en situación abdominal, de un $63 \%$ si es inguinal y de un $28 \%$ en testes normotópicos ${ }^{11}$. El pronóstico dependerá del estadio inicial y la histología del tumor.
La existencia de testículos no descendidos en la población adulta es rara en los países desarrollados, ya que en estos pacientes existe la norma de practicar orquidopexia electiva antes de los 2 años de vida, como forma de prevenir el cáncer y la infertilidad. La orquidopexia no elimina el riesgo de cáncer sino que permite un diagnóstico precoz al estar el testículo accesible a la exploración ${ }^{6,8}$.

Hay muchos estudios publicados sobre criptorquidia y el cáncer en testículos no descendidos. Sin embargo, no existen largas series de casos clínicos de tumores testiculares abdominales publicados en la literatura. La mayoría son casos clínicos aislados. Existen aproximadamente 42 casos clínicos de Seminomas en testículos abdominales publicados en revistas indexadas por Pubmed. La mayoría ente la década de los 50 y 80 . Luego con los avances en la investigación acerca de la evolución de testículos no descendidos, su relación con le cáncer y las formas de prevención, se publican menos casos. En los últimos 20 años hay cada vez menos casos publicados, lo que se explicaría por el progresivo descenso de la incidencia de los tumores en testículos abdominales debido a la prevención.

Como conclusión podemos decir que a pesar del riesgo elevado de cáncer testicular en pacientes con testículos intraabdominales, estimamos que esta corresponde a una patología de baja incidencia. Esto es debido la práctica regular de orquidopexia en pacientes prepuberales y la orquiectomía en los pacientes postpuberales con criptorquidia. Sin embargo, aún aparecen ocasionalmente algunos casos de tumores de testículos abdominales, que podrían ser evitados con la adecuada práctica de medidas de prevención.

\section{REFERENCIAS}

1. Batata MA, Whitmore JR WF, Chu FCH, Hilaris BJ, Loh J, Grabstald $\mathrm{H}$ et al. Cryptorchidism and testicular cancer. J Urol. 1980;124(3):382-387.

2. Kulkarni JN, Kamat MR. Tumors in undescended testis. J Surg Oncol 1991;46(4):257-260.

3. Rozanski TA, Bloom DA. The undescended Testis. Theory and Management. Urol Clin North Am 1995;22(1):107-118.

4. Villavicencio H, Martinez E, Vicente-Carretero R. Tumor testicular bilateral simultáneo. Actas Urol Esp 1989;13(5): 403-404. 
5. Gil Sanz MJ, Cebrian CC, Villanueva Benedicto A, Liedana Torres JM, Gonzalez Enguita C, Urruchi Fernandez P, et al. Manejo diagnóstico y terapéutico de los tumores testiculares. Revisión de 15 años. Arch Esp Urol 1991;44(4):417-423.

6. Abratt RP, Reddi VB, Sarembok LA. Testicular Cancer and Cryptorchidism. Br J Urol 1992;70(6):656-659.

7. Giwercman A, Grinsted J, Hansen B, Jensen OM, Skakkebaek NE. Testicular Cancer risk in boys with maldescended testis. A cohort study. J Urol. 1987;138(5): 12141216.

8. Rogers E, Teahan S, Gallagher H, Butler MR, Gringer R, McDermott TE, et al. The role of ochiectomy in the management of postpuberal cryptorchidism. J Urol. 1998;159(3): 851-854.

9. Cortes D, Thorup JM, Lenz K, Beck BL, Nielsen OH. Laparoscopy in 100 consecutive patientes with 128 impalpable testis. Br J Urol. 1995;75(3):281-287.
10. Brown IR, Dunlap HJ, Nizalik E, Schillinger JF. A child with an intra-abdominal testicular teratoma: a case report and review of prepuberal cryptorchid germ cell tumors. Urology 1995;46(6):863-866.

11. Carmona Campos E, Regueiro Lopez JC, Prieto Castro R, Leva Vallejo M, Moreno Arcas P, Requena Tapia MJ. Criptorquidia y Cancer testicular. Actas Urol. Esp 2000; 24(1):49-51.

Correspondencia autor: Dr. C. Cristián Palma.

Servicio de Urología del Hospital Clínico Universidad de Chile y

Hospital San José. Santiago, Chile.

E-mail autor: palmaceppi@hotmail.com

Información artículo: Nota clínica

Trabajo recibido: mayo 2006

Trabajo aceptado: septiembre 2006 